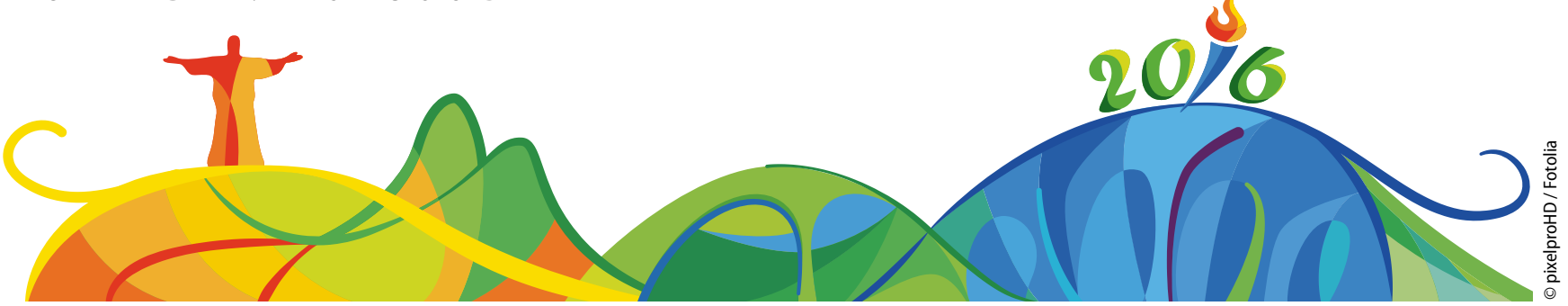

\title{
Welche Infektionsrisiken kommen auf die Sportfans in Brasilien zu?
}

\section{Wer sich trotz Zikavirus und beun- ruhigender Sicherheitshinweise zu einer Brasilienreise entschließt, um die Olympischen Spiele zu erleben, der sollte im Vorfeld gut beraten sein.}

\footnotetext{
_ Massenveranstaltungen sind immer Brutstätten verschiedenster Krankheitserreger. Findet das Großereignis in einer Region statt, die ohnehin verstärkt mit Gesundheitsproblemen zu kämpfen hat, steigt die Unsicherheit bei den Besuchern. Das European Centre for Disease Prevention and Control (ECDC) hat jetzt die Gesundheitsrisiken für Besucher der Olympiade 2016 zusammengefasst.

Nach Einschätzung der Infektionsexperten besteht für Sportfans in erster Linie die Gefahr gastrointestinaler Erkrankungen, etwa durch multiresistente Enterobacteriaceae über verschmutztes Wasser oder nicht ausreichend gegartes Fleisch. Bei Reiserückkehrern wurden als häufigste Verursacher anhaltender
}

Magen-Darm-Probleme Campylobacter und Giardia spp. nachgewiesen. Da zum Zeitpunkt der Spiele in Rio de Janeiro kühles und trockenes Klima herrscht, ist das Risiko für Krankheiten wie DengueFieber oder Infektionen durch Zikaoder Chikungunyaviren, die von Insekten übertragen werden, eher gering. Dennoch wird auch während dieser Zeit konsequenter Mückenschutz empfohlen.

\section{Höheres Risiko für Fußballfans}

Stärker gefährdet sind Besucher von Fußballspielen. Der Norden und Nordosten Brasiliens, darunter Manaus und Salvador, wo Endrundenspiele ausgetragen werden, gelten als Risikoregionen für ZikavirusInfektionen. Teile Brasiliens sind zudem Gelbfieber-Regionen, u. a. die Fußballorte Manaus, Belo Horizonte und Brasilia.

Das ECDC, das Auswärtige Amt und die Deutsche Gesellschaft für Tropenmedizin und Internationale Gesundheit raten Schwangeren und Frauen, die schwanger werden wollen, von Reisen in Zikavirus-Gebiete $a b$, da das Risiko frühkindli- cher Fehlbildungen bei einer Infektion nicht auszuschließen sei. Da sich gezeigt hat, dass das Virus sexuell übertragbar ist, wird männlichen Reiserückkehrern für die Dauer von zwei Monaten der Gebrauch von Kondomen empfohlen.

\section{Wer sich aus Rio hinausbewegt, sollte gewappnet sein}

Brasilienreisenden, die mehr vom Land sehen möchten, raten die Infektiologen, nicht nur die aktuelle Wirksamkeit ihrer Standardimpfungen inkl. Hepatitis-AImpfung zu überprüfen, sondern je nach Zielgebiet außerhalb von Rio auch eine Malariaprophylaxe zu erwägen. Wegen der Tollwutgefahr durch streunende Hunde und Katzen sollte bei Reisen durch ländliche Gebiete ggf. auch an eine Tollwutimpfung gedacht werden. Da die Olympiade in der Grippe-Hochsaison der südlichen und südöstlichen Hemisphäre stattfindet, könnte auch eine Influenzaimpfung angebracht sein. Typhus und Paratyphus sind selten, und seit 2005 gilt Brasilien als cholerafrei. -

Dr. Christine Starostzik

- Quelle: European Centre for Disease Prevention and Control. Potential risks to public health related to communicable diseases at the Olympics and Paralympics Games in Rio de Janeiro, Brazil 2016. Stockholm, 2016.

- Merkblatt des Auswärtigen Amtes für Reisende zu den Olympischen Spielen: http://www.brasil.diplo.de/contentblob/ 4827332/Daten/6624364/Gesundheit_Olympia_Rio.pdf

\section{Neues FSME-Risikogebiet in Deutschland}

In Bayern ist zu den bestehenden Risikogebieten für die Frühsommer-Meningoenzephalitis (FSME) 2016 der Landkreis Dachau hinzugekommen. Weiterhin besteht in Deutschland ein Risiko insbesondere in Bayern und Baden-Württemberg, Südhessen und im südlichen Thüringen, außerdem im mittelhessischen Landkreis Marburg-Biedenkopf, dem Saar-Pfalz-Kreis, dem Landkreis Birkenfeld und seit 2014 dem sächsischen Vogtlandkreis. Insbesondere im süddeutschen Raum dehnen sich die Risikogebiete langsam aus. Vereinzelte FSME-Fälle wurden in den letzten Jahren aber in fast allen Bundesländern beobachtet. Allen zeckenexponierten Personen in Risikogebieten empfiehlt die STIKO die FSME-Impfung.

- Quelle: RKI. Epidemiologisches Bulletin Nr. 18; 9. Mai 2016

\section{Impflinge morgens einbestellen}

Vor allem ältere Patienten profitieren möglicherweise davon, wenn sie in die Morgensprechstunde bestellt werden, um ihren Impfstatus auf den aktuellen Stand zu bringen. In einer britischen Studie in 24 Allgemeinarztpraxen hat sich gezeigt, dass Patienten, die ihre jährliche Grippeimpfung zwischen neun und elf Uhr erhalten hatten, einen Monat nach der Impfung signifikant mehr Antikörper gegen H1N1 sowie Influenza B gebildet hatten als diejenigen, die ihre Spritze erst am Nachmittag bekommen hatten. Antikörper gegen H3N2 sowie Zytokin- und Steroidhormonspiegel blieben von dieser Variante unbeeinflusst. Teilnehmer der Studie waren 276 immunkompetente Senioren ab 65 Jahren.

- Quelle: Long J E et al. Vaccine 2016;34:2679-2685 